\title{
AVALIAÇÃO E INTERVENÇÃO PSICOPEDAGÓGICAS: UM ESTUDO DE CASO DE UMA CRIANÇA EM IDADE ESCOLAR COM HIPÓTESE DE DISLEXIA
}

\author{
PSYCHOPEDAGOGICAL EVALUATION AND INTERVENTION: A CASE \\ STUDY OF A SCHOOL-AGE CHILD WITH DYSLEXIA HYPOTHESIS
}

\author{
Sebastião Gomes Barbosa \\ Especialista em Psicopedagogia Clínica e Institucional \\ Universidade Estácio de Sá - UNESA. \\ Rio de Janeiro, RJ - BR \\ sgomes2013@hotmail.com
}

\begin{abstract}
Resumo: A dislexia é um transtorno neurobiológico que compromete habilidades de leitura e escrita e tem repercussão por toda a vida do sujeito disléxico. Este artigo refere-se a um caso de uma criança com 8 anos e 4 meses de idade, do sexo masculino e do terceiro ano de escolaridade, com hipótese de dislexia. O objetivo desse estudo foi apresentar a importância da avaliação e intervenção psicopedagógicas na recuperação da dislexia. A abordagem da pesquisa é de ordem qualitativa e foi desenvolvida por meio de estudo descritivo. A avaliação psicopedagógica foi importante para a hipótese diagnóstica de dislexia. Após a intervenção psicopedagógica, houve diminuição nos efeitos do transtorno e a criança melhorou significativamente na leitura, escrita e aritmética. Este estudo demonstrou a importância da avaliação e intervenção psicopedagógicas no caso de recuperação da dislexia.
\end{abstract}

Palavras-chave: avaliação psicopedagógica; dificuldades de aprendizagem; dislexia; intervenção psicopedagógica.

\begin{abstract}
Dyslexia is a neurobiological disorder that compromises reading and writing skills and reverberates throughout the entire life of the dyslexic person. This article discusses the case of a male 8-year-and-4months-old child enrolled in the third year of primary school, with a dyslexia hypothesis. The study aimed to present the importance of psychopedagogical assessment and intervention during the dyslexia recovery process. This article had a qualitative research approach, and it was developed through a descriptive study. From the psychopedagogical assessment performed, there was a reduction in the effects of the disorder, and the child displayed significant improvement in his reading, writing, and arithmetic skills. This study demonstrated the importance of psychopedagogical assessment and intervention in the dyslexia recovery case.
\end{abstract}

Keywords: psychopedagogical evaluation; learning difficulties; dyslexia; psychopedagogical intervention.

\section{Para citar - ABNT NBR 6023:2018}

BARBOSA, Sebastião Gomes. Avaliação e intervenção psicopedagógicas: um estudo de caso de uma criança em idade escolar com hipótese de dislexia. Cadernos de Pós-graduação, São Paulo, v. 20, n. 1, p. 203-217, jan./jun. 2021. Disponível em: https://doi.org/10.5585/cpg.v20n1.19439. 


\section{Introdução}

Este artigo tem como objetivo evidenciar a importância da avaliação e intervenção psicopedagógicas em um caso de hipótese de dislexia. Trata-se de um estudo de caso sobre uma criança com dificuldades de leitura, escrita e aritmética que frequenta uma escola pública na Rede Municipal de Ensino de Nova Iguaçu, no Estado do Rio de Janeiro. A criança foi levada a clínica psicopedagógica pela mãe por solicitação da escola, pois não avançava nos processos de aprendizagem de leitura, escrita e aritmética, apesar de o aluno frequentar o projeto de reforço escolar, no contraturno da escola, e da intervenção da professora em sala de aula.

A abordagem para este caso específico será realizada no campo da Psicopedagogia, buscando compreender qual a relação que o aprendente estabelece com a aprendizagem e quais os vínculos envolvidos nesse processo. De acordo com o capítulo 1 , artigo $1^{\circ}$, do Código de Ética do Psicopedagogo da Associação Brasileira de Psicopedagogia (ABPp), o campo de conhecimento de atuação da Psicopedagogia é interdisciplinar, envolvendo tanto a Educação como a Saúde; e ainda, segundo o referido código, na intervenção psicopedagógica não são dissociáveis os processos de aprendizagem das dificuldades e possibilidades dos sujeitos e sistemas (ASSOCIAÇÃO BRASILEIRA DE PSICOPEDAGOGIA, 2019).

É nesse quadro de complexidade do conhecimento que a Psicopedagogia vem se constituindo como terreno fértil na busca para compreender e atender o ser humano de forma integral, rompendo com a visão fragmentada. Nessa perspectiva, uma intervenção psicopedagógica bemsucedida deve ter antes uma avaliação cuidadosa, na qual o sujeito deve ser contemplando de forma integral. O desenvolvimento do olhar e da escuta psicopedagógicas são fundamentais para atingir esse objetivo. Munhoz (2004), citando Moran (2002), defende uma abordagem psicopedagógica que considere a aprendizagem como um fenômeno complexo no qual as interações e interligações possam estar presentes.

Diante do exposto, é importante identificar os aspectos relacionados às dificuldades de aprendizagem para realizar a intervenção adequada (WEISS, 2000; BOSSA, 2000; RUBINSTEIN, 2000; CASTANHO, 2018).

\section{Metodologia}

O trabalho foi desenvolvido por meio de estudo de caso, pois buscou descrever o percurso da avaliação e intervenção psicopedagógicas na investigação das dificuldades de aprendizagem a partir de variadas fontes: entrevista semiestruturada, instrumentos de avaliação específicos e interações informais com os sujeitos do caso, com o intuito de obter maior profundidade de análise, a partir do máximo de informações importantes. Em consonância com o método de estudo de caso, 
utilizou-se a abordagem qualitativa. Realizou-se o processo de investigação e intervenção psicopedagógicas na clínica Suzana Assunção Casanova, localizada em Belford Roxo-RJ. Com a finalidade de esclarecer como foram desenvolvidos os procedimentos com a criança/aprendente, o método deste estudo foi dividido da seguinte maneira: 1) Avaliação psicopedagógica 2) Intervenção psicopedagógica.

O sujeito da pesquisa é uma criança do sexo masculino, de 8 anos e 4 meses de idade, matriculada no terceiro ano do ensino fundamental em uma escola pública do município de Nova Iguaçu-RJ. Foi encaminhado pela orientadora pedagógica da escola para o atendimento psicopedagógico com queixa de dificuldades em leitura, escrita e aritmética, pois, apesar de reforço escolar, não avançava na aprendizagem. Os pais da criança assinaram um Termo de Consentimento Livre Esclarecido para o prosseguimento da pesquisa. Para preservar a identidade da criança, será chamada de W, conforme prevê o TCLE em pesquisas com seres humanos. Em outros momentos no texto, serão utilizadas as palavras "criança/aprendente".

A avaliação psicopedagógica foi realizada a partir das inter-relações família-aprendente-escola, buscando compreender como os aspectos relacionados aos campos familiar e escolar, além dos aspectos relacionados a própria criança, podem interferir na aprendizagem. Os instrumentos específicos de avaliação (Entrevista Operativa Centrada na Aprendizagem/EOCA, Teste de Desempenho Escolar/TDE, Provas Operatórias, Técnicas Projetivas, anamnese), foram utilizados com o objetivo de investigar aspectos relacionados à aprendizagem e os vínculos estabelecidos com o conhecimento. Quanto à opção por entrevistas semiestruturadas, a finalidade foi compreender aspectos da vida da criança/aprendente no ambiente familiar e da escola com mais profundidade. A realização das entrevistas possibilitou um olhar amplo sobre questões que necessitavam de mais informações ou esclarecimentos.

Para avaliação psicopedagógica, as sessões aconteceram uma vez por semana, com duração de 50 minutos cada. Finalizada a avaliação, e com os resultados analisados, foi realizada uma entrevista devolutiva com os pais, comunicando suscintamente todo o processo de avaliação e os aspectos que interferiam na problemática de aprendizagem. No que se refere à escola, algumas estratégias relacionadas às avaliações, conteúdos e atividades foram combinadas com a equipe pedagógica e professora da criança, com o intuito de auxiliá-la na vida escolar.

O início das intervenções psicopedagógicas aconteceu após acordo com os pais sobre o número de sessões e quantidade de horas, sendo estabelecido que cada sessão duraria 1 hora e 30 minutos. No total, ocorreram 112 sessões de intervenção e elas foram realizadas a partir de diferentes ferramentas, a saber: intervenção de base fonológica, treinamento das habilidades visuomotoras, intervenção por meio de tecnologia computadorizada, intervenção com base socioemocional 
(autoestima), intervenção no campo da matemática com base no pensamento operacional concreto (CUNHA, 1976). A partir dessas ferramentas, foram disponibilizadas atividades lúdicas, jogos, histórias infantis, músicas e brincadeiras, com o objetivo de trabalhar na recuperação da dislexia, observando a característica da criança e o seu nível pedagógico.

\section{O campo da psicopedagogia e as dificuldades de aprendizagem}

Quando se fala em dificuldades de aprendizagem escolar, é preciso entender que não se trata apenas da dificuldade de os alunos aprenderem determinados conteúdos, ou de não desejarem estudar ou, ainda, de lhes faltarem competências e habilidades para isso. Trata-se de uma questão ampla e profunda, cujas causas precisam ser investigadas com cuidado. Fernández (1991) usa o termo "inteligência aprisionada" para se referir a problemática do não-aprender, além de levantar questões importantes que precisam ser pensadas na clínica psicopedagógica e nos espaços de docência, tais como: trabalho alegre, sereno e entusiasta e o lugar do aprender como algo prazeroso.

Trazendo o desvio de aprendizagem sob o enfoque escolar, familiar e social, Weiss (2011) defende um caminho de investigação psicopedagógica amplo, no qual os bloqueios de aprendizagem escolar possam ser descobertos. Sob a ótica de Bossa (2002), a psicopedagogia tem um papel muito importante no desenvolvimento e aprendizagem do sujeito com dificuldades de aprendizagem, e a escola deve enfrentar a problemática das dificuldades de aprendizagem a partir da multiplicidade do conhecimento humano, rompendo com o fracasso escolar.

Traçando um caminho parecido, Castanho (2018) defende a psicopedagogia como um campo multidisciplinar e interdisciplinar no qual a aprendizagem corresponda à realidade dos tempos pós-modernos, marcados pela complexidade da dimensão humana. Esses estudos no campo da psicopedagogia apontam para mudanças necessárias na maneira de a escola lidar com questões ligadas ao ensino-aprendizagem, ao fracasso escolar e ao papel da psicopedagogia e da escola no enfrentamento dessas questões.

No âmbito escolar, especificamente, os obstáculos na aprendizagem podem ser gerados ou agravados de acordo com a maneira como a escola concebe o conhecimento e norteia suas ações. Uma escola que supervaloriza os conhecimentos cognitivos e trata a todos dentro de um único padrão, por exemplo, não favorece a aprendizagem de todos. Embora a escola possa gerar ou agravar as dificuldades de aprendizagem, quando há uniformização e padronização nos aspectos pedagógicos, uma vez que não há um único tipo de conhecimento e nem um único tipo de aluno, muitos outros aspectos também podem interferir na aprendizagem.

Como menciona Parente e Ranña (1987) as dificuldades de aprendizagem fazem parte de um sistema complexo que pode envolver aspectos culturais, sociais, pedagógicos, psicológicos e 
médicos. O psicopedagogo precisa levar isso em consideração, sob pena de equivocar-se ou alienarse na investigação do que está interferindo na aprendizagem (PARENTE; RANÑA, 1987). Dito isso, será apresentado a seguir a esquematização da avaliação do aprendente com o percurso realizado para encontrar a origem das dificuldades de leitura, escrita e aritmética.

\section{Avaliação: instrumentos e procedimentos utilizados}

O processo de avaliação foi realizado a partir das inter-relações família-aprendente-escola. Para isso, instrumentos específicos e sessões com tempo estabelecido foram organizados, conforme especificado na Tabela 1.

Tabela 1 - Quantidade de sessões e instrumentos de avaliação

\begin{tabular}{|c|c|c|c|}
\hline Sujeitos & $\mathrm{N}^{\circ}$ de sessões & Duração das sessões & $\begin{array}{c}\text { Instrumentos utiliza- } \\
\text { dos }\end{array}$ \\
\hline Mãe & 01 & $50 \mathrm{~min}$ & Entrevista inicial \\
\hline \multirow[t]{4}{*}{ Aprendente } & 06 & $50 \mathrm{~min}$ & EOCA \\
\hline & & $50 \mathrm{~min}$ & $\begin{array}{l}\text { Teste de Desempenho } \\
\text { escolar (TDE) }\end{array}$ \\
\hline & & $2 \mathrm{~h} 30 \mathrm{~min}$ & $\begin{array}{c}\text { Provas Operatórias (Pi- } \\
\text { aget) }\end{array}$ \\
\hline & & $50 \mathrm{~min}$ & Técnicas Projetivas \\
\hline Pai e mãe & 01 & $50 \mathrm{~min}$ & $\begin{array}{c}\text { Anamnese (ficha com } \\
\text { entrevista) }\end{array}$ \\
\hline Pai & 01 & $50 \mathrm{~min}$ & Entrevista \\
\hline \multirow[t]{2}{*}{ Professora } & 01 & $50 \mathrm{~min}$ & Entrevista \\
\hline & 01 & $50 \mathrm{~min}$ & Entrevista \\
\hline TOTAL & 11 & $9 \mathrm{~h} 10 \mathrm{~min}$ & 09 \\
\hline
\end{tabular}

Fonte: Elaborado pelo autor.

Além desses instrumentos avaliativos, foram levados em consideração, durante o processo investigativo das dificuldades de aprendizagem, os relatos verbais do pai, da mãe, da professora e da própria criança.

\section{História de vida precedente e da atualidade}

Dados físicos, emocionais, ambientais e escolares

A partir da análise da ficha de anamnese ${ }^{1}$ preenchida pelos pais e das entrevistas, foi possível perceber que a gestação de W ocorreu aparentemente sem nenhum acontecimento que interferisse no desenvolvimento. O parto foi natural e no tempo previsto. Foi amamentado até os seis meses, 
depois passou a alimentar-se com alimentos sólidos. Em relação ao desenvolvimento psicomotor, demorou para sentar-se e andar. Segundo o pai, parecia "mais mole", quando começou a sentar, do que as outras crianças e andou com quase dois anos de idade. No que se refere à linguagem, demorou a falar, pronunciando as primeiras palavras por volta dos três anos de idade.

De acordo com o pai, W falava poucas vezes e com frases curtas para atender necessidades básicas imediatas (fome, sede, necessidade de ir ao banheiro) ou quando queria colo. A mãe de W acrescentou que ele também trocava com frequência os nomes dos objetos de casa. A relação entre pais e filho, tanto em casa como em outros ambientes, demonstra ser afetuosa. W é oriundo de uma família com bom nível socioeconômico.

Quanto à escolarização, W estuda em uma escola que utiliza métodos tradicionais. As dificuldades de W já eram percebidas desde a Educação Infantil, sendo sinalizado pela professora nos relatórios descritivos individuais da vida escolar. É possível perceber, por exemplo, as dificuldades para acompanhar o ritmo das músicas, recontar uma história ouvida e identificar rimas. No primeiro ano do ciclo de alfabetização, as dificuldades ficaram ainda mais evidentes, pois $\mathrm{W}$ demorava mais do que o normal para copiar as atividades do quadro e a escrita apresentava os traçados das letras de forma irregular, com letras ora muito grandes ora muito pequenas. Além disso, havia dificuldade em utilizar a orientação espacial no caderno no momento da escrita.

Havia algum grau de comprometimento nas habilidades de discriminação dos sons das palavras e na correspondência aos símbolos gráficos (letras), assim como nos símbolos matemáticos. Também apresentava dificuldades na memorização e organização das operações matemáticas. Devido à demora de $\mathrm{W}$ para escrever e aos erros ortográficos, quando copiava alguma atividade do quadro, ou respondia alguma atividade de matemática, a professora, a princípio, pensou que ele tivesse alguma dificuldade para enxergar, solicitando a mãe que o levasse ao oftalmologista. No entanto, o exame oftalmológico demonstrou que não havia nenhum problema na visão.

No segundo ano do ciclo de alfabetização, as dificuldades anteriores persistiram durante todo o ano letivo. Então, W foi aprovado para o terceiro ano de escolaridade com muitas demandas relacionadas à leitura, escrita e aritmética com as quais precisava lidar. Nesse período, ele demonstrava desânimo e vontade de desistir da escola por não acompanhar os colegas nas atividades propostas. Outros aspectos importantes da vida escolar de W foram observados nos relatos da professora, conforme o percurso apontado por Baroja, Paret e Riesgo (1978): 
- Quanto à interação da criança no grupo: interage bem com os colegas da turma no recreio. Entretanto, tem se mostrado mais quieto do que o normal, isolando-se um pouco das brincadeiras.

- A adequação ao ambiente escolar: demonstra bom comportamento com todas as pessoas, assim como em todos os ambientes de participação escolar (recreio, cantina, pátio, corredores, biblioteca etc.)

- O seu desempenho em relação à opinião que a professora tem da sua capacidade, fundamentalmente no que se refere à leitura e escrita: mostra-se envergonhado devido ao baixo desempenho. No entanto, gosta da professora, diz que ela é paciente e carinhosa.

- Existência de alguma anomalia observada pela professora: mostrava-se bastante desorganizado nas atividades e materiais de estudo.

A partir do contexto, a orientadora pedagógica da escola chamou a mãe de W para conversar sobre a vida escolar dele e uma possível participação no projeto de reforço escolar, que era ofertado no contraturno da escola. O resultado esperado no projeto, porém, não foi alcançado, pois não houve avanços no que diz respeito à leitura, escrita e aritmética. A orientadora pedagógica, então, fez o encaminhamento escolar para que a mãe procurasse um profissional especializado para o caso, uma vez que, apesar de todos os esforços da escola, o problema persistia. Nesse período, W encontrava-se desmotivado com os estudos, devido às dificuldades de aprendizagem, procurando desculpas para não ir à escola, acordando mais tarde ou reclamando de cansaço excessivo, segundo relatos da mãe.

\section{Avaliação específica solicitada}

Para verificar possíveis interferências de problemas auditivos nas dificuldades de aprendizagem da leitura e escrita, foi solicitado o encaminhamento para um fonoaudiólogo. Os resultados dos do exame de audiometria indicaram que não havia deficiência auditiva.

\section{A intervenção psicopedagógica}

Para evitar que a intervenção sobre a problemática das dificuldades de aprendizagem caísse no reducionismo, fez-se a investigação psicopedagógica a partir de múltiplos aspectos em três situações: a família, o aprendente e a escola. A escola é uma microssociedade na qual a criança precisará aprender a conviver com as diferenças e as divergências do grupo. A aproximação entre ela 
e a família favorece a discussão sobre pontos importantes da vida escolar, que são fundamentais para o êxito do ensino-aprendizagem (PORTELLA; HICKEL, 2010). Vai-se à escola para aprender, para compartilhar do conhecimento historicamente produzido e para socializar. Nessa perspectiva, não somente a família se beneficia, mas a sociedade em geral, quando a escola desempenha bem o seu papel. Todavia, quando há fracasso escolar de um aluno, a escola de certa forma também fracassa.

O esquema da Figura 1 mostra a inter-relação família-aprendente-escola como componentes imprescindíveis para o progresso no ensino-aprendizagem.

Figura 1 - Intervenção psicopedagógica

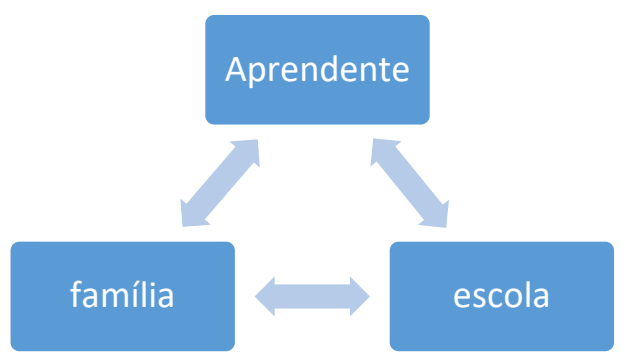

Fonte: Elaborado pelo autor.

Segundo Munhoz (2004), a Psicopedagogia deve ser pensada a partir de uma visão sistêmica, na qual a velha ciência tradicional perde espaço para uma ciência mais aberta e ampla, condizente com a contemporaneidade. Isso significa pensar o conhecimento e a aprendizagem como uma rede complexa onde todos os elementos estão interligados.

Nesse sentido, aprender não é um ato solitário. Tanto o sujeito da aprendizagem quanto os ensinantes, neste caso a escola e a família, também aprendem (MUNHOZ, 2004). Diante desse contexto, é importante esclarecer que as intervenções foram realizadas no ambiente da clínica psicopedagógica, a partir da visão sistêmica. Foram organizadas 75 sessões, conforme esquematizado na Tabela 2. Optou-se por cinco tipos de intervenção na recuperação da dislexia, sempre enfatizando o aspecto lúdico. 
Tabela 2 - Proposta psicopedagógica de recuperação da dislexia

Ferramentas de intervenção $\quad \mathbf{N}^{\circ}$ de sessões Quantidade de horas e minutos

\begin{tabular}{lcc}
\hline $\begin{array}{l}\text { Intervenção de base fonológica } \\
\begin{array}{l}\text { Treinamento das habilidades visuo- } \\
\text { motoras }\end{array}\end{array}$ & 20 & $30 \mathrm{~h}$ \\
$\begin{array}{l}\text { Intervenção por meio de tecnologia } \\
\text { computadorizada }\end{array}$ & 15 & $22 \mathrm{~h} 30 \mathrm{~min}$ \\
$\begin{array}{l}\text { Intervenção com base socioemocio- } \\
\text { nal (autoestima) }\end{array}$ & 5 & $22 \mathrm{~h} 30 \mathrm{~min}$ \\
$\begin{array}{l}\text { Intervenção no campo da matemática } \\
\text { com base no pensamento operacional } \\
\text { concreto (Piaget) }\end{array}$ & 20 & $7 \mathrm{~h} 30 \mathrm{~min}$ \\
\hline
\end{tabular}

TOTAL

Fonte: Elaborado pelo autor.

Resultados

Alinhado ao que foi indicado no campo metodológico, os resultados da pesquisa serão apresentados da seguinte maneira: 1) Resultados da avaliação psicopedagógica 2) Resultados da intervenção psicopedagógica.

\section{Resultados da avaliação psicopedagógica}

No Teste de Desempenho Escolar (STEIN, 1994), foi possível perceber que as áreas de leitura, escrita e aritmética se encontram prejudicadas, com Escore Bruto Total abaixo do esperado para idade cronológica e escolar.

No que se refere às provas piagetianas ${ }^{2}$ (WEISS, 2000), apresentou condutas intermediárias (Nível 2) - oscilação entre conservação e não-conservação no que tange à conservação de quantidade e a conservação de comprimento. Na prova de quantificação, foram utilizados os seguintes critérios: cores, formas e tamanhos, sem dificuldade de raciocínio (Nível 3). No entanto, respondeu às perguntas de intersecção e inclusão ora corretamente, ora de forma errônea, demonstrando um nível intuitivo articulado (Nível 2).

Na EOCA (VISCA, 1987), demonstrou criatividade, embora com pouca iniciativa. Apresentou lentidão na execução das atividades livres. Nas técnicas projetivas (VISCA, 2008), o Teste do Par Educativo mostrou a escola distante na visão do aprendente. A professora, porém, aparece sorridente e acolhedora. Em relação ao teste da Família, o vínculo é positivo. O aprendente aparece 
feliz em um dia de sol durante um passeio na praia com os pais. O Teste Livre mostra o futebol como sua brincadeira favorita.

Em relação ao que foi registrado na ficha de anamnese, percebeu-se, na história de vida precedente e atual, que havia atrasos no desenvolvimento psicomotor e na linguagem. No que diz respeito à vida escolar, as dificuldades de aprendizagem podiam ser percebidas desde a educação infantil. Dificuldades estas que foram ficando mais acentuadas nos anos seguintes, durante a fase de alfabetização, devido às demandas escolares com a leitura, escrita e aritmética.

A partir da aplicação e interpretação dos resultados dos instrumentos avaliativos anamnese (ficha com entrevista), EOCA, Técnicas Projetivas, provas piagetianas, entrevista com o pai e a mãe (em conjunto), entrevista com o pai e a mãe (individualmente), entrevista com a professora, Teste de Desempenho Escolar (TDE), foi possível perceber, por exclusão, que as dificuldades de leitura, escrita e aritmética do aprendente estão relacionadas a um transtorno específico de aprendizagem, mais especificamente a dislexia.

Como foi constatado, não há deficiências visuais e auditivas. Também não há problemas pedagógicos escolares geradores de problemas de leitura, escrita e aritmética (embora a escola utilize métodos convencionais de ensino que podem ter ampliado esses problemas). A criança não faz parte de uma família disfuncional (WAGNER, 2002), o que também poderia interferir no processo de leitura, escrita e aritmética.

Os relatórios individuais descritivos da escola mostraram que, desde a Educação Infantil, já havia sinais de dificuldades na aprendizagem, como dificuldade para acompanhar o ritmo das músicas infantis, identificar as rimas e contar uma história. As dificuldades foram aumentando posteriormente com as demandas exigidas no $1^{\circ}$ e $2^{\circ}$ anos de escolaridade. No $3^{\circ}$ ano de escolaridade, as dificuldades de leitura escrita ficaram mais evidentes, uma vez que as demandas escolares se intensificaram.

No final de toda a avaliação, foi comunicado uma devolutiva aos pais explicando que as dificuldades de W na leitura, escrita e aritmética, sinalizadas pela escola, estão relacionadas ao transtorno específico de aprendizagem conhecido como dislexia, e que a origem desse transtorno é neurobiológica. Foi dito que o transtorno compromete áreas específicas da pessoa correspondentes às habilidades de ler e escrever. Foi comunicado que algumas pessoas com dislexia têm problemas com aritmética (soma, subtração, multiplicação e divisão) e outros aspectos dessa área, e que esse é o caso de W.

Foi informado que, mesmo sendo inteligente, a pessoa disléxica pode ter dificuldade em reconhecer letras e associar o som à grafia/escrita das palavras e não por preguiça de realizar as atividades escolares ou má vontade de aprender. Foi comunicado também, de forma suscinta e 
objetiva, como aconteceram os procedimentos adotados na investigação da problemática da aprendizagem, enfatizando, como aponta Weiss (2000), os aspectos positivos.

Isso é muito importante, porque é comum que a pessoa com dislexia escute muitas palavras negativas das pessoas no seu cotidiano, que as julgam preguiçosas ou desleixadas. Geralmente, os pais chegam à clínica com um olhar de desesperança por não terem encontrado uma solução fácil e rápida para os problemas dos filhos. Muitas das vezes, veem a clínica psicopedagógica como uma "tábua de salvação". Foi esclarecido que a dislexia é uma condição permanente, porém, com as intervenções adequadas, os efeitos relacionados ao transtorno são atenuados. Dito isso, é importante destacar que, ao finalizar o comunicado aos pais, eles pareciam mais tranquilos e confiantes em relação à superação das dificuldades de W na vida escolar e pessoal. Os pais foram esclarecidos quanto à necessidade da intervenção psicopedagógica para a recuperação da dislexia e da dificuldade de matemática associada.

Foram, então, acordados o número de sessões na clínica e a quantidade de horas para as intervenções na dislexia. Foram transmitidas orientações e recomendações aos pais em relação à maneira de como lidar com as dificuldades do filho. Importante destacar que a avaliação psicopedagógica foi imprescindível para a tomada de decisão referente à proposta de intervenção psicopedagógica.

A partir da hipótese diagnóstica de dislexia de W, e de elementos associados com o transtorno, como a baixa autoestima, a lentidão na execução das tarefas, o baixo desempenho escolar, dentre outros elementos, foram realizadas análises para encontrar a melhor maneira de recuperação da dislexia, de acordo com as especificidades e potencialidades de W. Diante desse contexto, atividades lúdicas e jogos, dentre outras, sob o enfoque de diferentes ferramentas interventivas, foram realizadas e foi possível observar interesse de $\mathrm{W}$ na realização das atividades propostas.

Quanto à escola, após a devolutiva, foram traçadas estratégias com a equipe pedagógica e professora, buscando melhorar aspectos relacionados às avaliações, conteúdos, atividades, dentre outros aspectos, necessários para reduzir os efeitos do transtorno de dislexia na vida escolar de W.

\section{Resultados da intervenção psicopedagógica}

Com a intervenção no campo fonológico, houve melhoras nas habilidades de leitura, uma vez que o aprendente ampliou o repertório de conhecimento de fonemas. Além disso, pode-se perceber também melhoras nos aspectos ortográficos relacionados à sua produção escrita. $\mathrm{O}$ treinamento de habilidades visumotoras contribuiu para uma melhor percepção visual e consequente melhora na condição de escrita: traçado das letras, memória visual, desenvolvimento perceptivo 
espacial. Com o uso de software específico para treino nas habilidades de leitura, pode-se perceber um ganho qualitativo na velocidade da leitura das palavras.

Em relação a intervenção com base socioemocional, percebeu-se maior autoconhecimento e aceitação das limitações do aprendente. A criança desenvolveu, nesse sentido, maior habilidade para lidar com as suas próprias emoções e a dos outros. No que diz respeito à área da matemática, os trabalhos com base no pensamento operacional concreto de Piaget (CUNHA, 1976), com jogos e atividades lúdicas diversificadas, como quebra-cabeça, dominó, jogos de regras envolvendo aritmética, bingo, dentre outros, melhoraram de forma significativa as situações relacionadas ao cálculo matemático. Importante destacar que o aprendente está progredindo para um raciocínio abstrato, apesar de seu raciocínio lógico matemático ainda estar inserido em uma realidade concreta.

As sessões de intervenção psicopedagógica foram realizadas com duração de 1 hora e 30 minutos cada, totalizando 112 horas e 30 minutos. De forma geral, as intervenções focadas nas especificidades do aprendente, levando em consideração suas capacidades e possibilidades, promoveram um avanço significativo no que se refere à leitura, escrita e aritmética. Foi possível observar que, à medida que as sessões com as intervenções psicopedagógicas foram acontecendo, o aprendente foi progredindo cada vez mais no desenvolvimento dessas habilidades.

\section{Discussão}

A queixa escolar, trazida pelos pais de W à clínica psicopedagógica, a respeito das dificuldades de aprendizagem da leitura, escrita e aritmética, pôde ser investigada no campo da Psicopedagogia. Relatos de dificuldades de aprendizagem são comuns nos Conselhos de Classe e nas falas dos professores no ambiente escolar. Tais dificuldades diferenciam-se dos transtornos específicos de aprendizagem. Não são todas as crianças com dificuldades de aprendizagem que apresentam transtornos ou distúrbios. O contrário, porém, é verdadeiro: crianças com algum tipo de transtorno ou distúrbio geralmente apresentam dificuldades no aprendizado.

Segundo Rubinstein (2000), o psicopedagogo assume um papel semelhante a um detetive na busca de pistas durante a investigação do processo de aprendizagem. Em um primeiro momento, algumas dessas pistas podem ser falsas ou sem importância, mas, ao final de toda a investigação, chega-se ao entendimento do que causa a dificuldade de aprendizagem. Para encontrar a origem das dificuldades de aprendizagem de W, foi necessária uma investigação ampla, que englobou não somente o próprio aprendente, mas as suas relações no contexto das instituições básicas em que estava inserido (WEISS, 2011). Nesse sentido, escola, família e aprendente, assim como suas inter-relações, foram tomados como parte do processo de investigação na clínica psicopedagógica. 
No entendimento de Bossa (2000), o psicopedagogo tem um papel muito importante na dinâmica da comunidade educativa, pois pode facilitar o processo de aprendizagem ao intervir nos obstáculos que possam atrapalhar. Dessa forma, o psicopedagogo pode ser um ponto de apoio para as famílias já fragilizadas com os obstáculos de aprendizagem e prejuízos escolares e sociais relacionados. Dito isso, as concepções de Rubinstein (2000), Bossa (2000) e Weiss (2011) foram fundamentais para entender a função da hipótese diagnóstica e da avaliação psicopedagógica, além de esclarecer procedimentos importantes que seriam utilizados nas investigações das dificuldades de aprendizagem de $\mathrm{W}$.

Após a identificação de elementos na vida escolar, pessoal e familiar de W que explicavam as dificuldades de aprendizagem, foi combinado com os pais as intervenções psicopedagógicas e o planejamento de como isso deveria acontecer. A hipótese de dislexia para o caso de $\mathrm{W}$ trouxe novas esperanças e expectativas para a família. O esclarecimento aos pais de que não havia cura para a dislexia, mas intervenções capazes de atenuar o comprometimento nas habilidades de leitura, escrita e matemática, trouxeram um novo olhar para a realidade, pois o caminho para superação exigiria esforço e persistência.

Signor, Claessen e Leitão (2020), apontam que sentimentos de incapacidade, baixa autoestima, depressão e vergonha podem ser identificados em pessoas com dislexia. No caso de W, foi preciso resgatar a autoestima através da valorização de seus esforços, e de elogios e destaques aos aspectos positivos. Houve avanços na aprendizagem de $\mathrm{W}$ após as intervenções psicopedagógicas.

Apesar dos bons resultados alcançados nas intervenções psicopedagógicas, o transtorno de dislexia continuará sendo um desafio para W durante toda a sua vida. Dessa forma, as orientações dadas à família e à escola precisam ser seguidas para que os obstáculos na aprendizagem de W possam ser superados em cada fase da vida escolar.

\section{Considerações finais}

Este estudo de caso trouxe à tona uma investigação e intervenção psicopedagógica das dificuldades de aprendizagem da leitura e escrita de uma criança em idade escolar, com base em instrumentos avaliativos específicos. A partir da avaliação psicopedagógica, percebeu-se que a hipótese das dificuldades de leitura e escrita é um transtorno específico de aprendizagem: a dislexia. Também foi observado que as dificuldades na aritmética, no caso de W, também estão associadas ao transtorno.

É possível perceber que as intervenções para atenuar as dificuldades de aprendizagem de W poderiam ter sido feitas desde muito cedo. Entretanto, os sintomas que indicavam que algo não estava bem no desenvolvimento e aprendizagem só foram levados em consideração pelos pais mais 
tarde, quando os prejuízos escolares se tornaram acentuados. O diagnóstico precoce da origem da problemática de aprendizagem, assim como a intervenção adequada, poderia evitar consideravelmente o sofrimento da criança e da família, pois quanto mais cedo o transtorno é descoberto, maior é a perspectiva de êxito na recuperação dos prejuízos.

Após a aplicação de ferramentas interventivas na recuperação da dislexia, no campo psicopedagógico, os efeitos do transtorno se amenizaram e foram observadas melhoras significativas na aprendizagem da leitura, escrita e aritmética. Nesse estudo de caso, ressalta-se a importância da avaliação e intervenção psicopedagógicas na recuperação da dislexia.

\section{Notas}

${ }^{1}$ Para evitar um olhar preconcebido sobre a vida do aprendente, a anamnese foi uma das últimas sessões realizadas.

${ }^{2}$ Nas provas piagetianas para evitar "contaminação" de respostas, foram realizadas 3 sessões em dias diferentes, com 50 minutos cada, totalizando 2 horas e 30 minutos.

\section{Referências}

ASSOCIAÇÃO BRASILEIRA DE PSICOPEDAGOGIA. Código de Ética. ABPPp, Recife, 26 out. 2019. Disponível em: https://www.abpppe.com.br/codigo-de-etica. Acesso em: 20 maio 2020.

BAROJA, Fernanda Fernández; PARET, Ana María Llopis; RIESGO, Carmen Pablo de. La Dislexia: origen, diagnostico e recuperacion. 4. ed. Madri, 1978.

BOSSA, Nadia A. Fracasso Escolar: um olhar psicopedagógico. São Paulo: Artmed, 2002.

BOSSA, Nádia A. Psicopedagogia no Brasil: contribuições a partir da prática. Porto Alegre: Artes Médicas Sul, 2000.

CASTANHO, Marisa Irene Siqueira. A psicopedagogia em um diálogo multidisciplinar. Psicopedagogia, São Paulo, v. 35, n. 106, p. 116-124, 2018. Disponível em http://pepsic.bvsalud.org/scielo.php?script=sci_arttext\&pid=S0103-84862018000100013. Acesso em: 4 nov. 2020.

CUNHA, Maria Auxiliadora Versiani. Didática fundamentada na teoria de Piaget. Rio de Janeiro: Forense-Universitária, 1976.

FERNÁNDEZ, Alicia. A Inteligência Aprisionada. Tradução Iara Rodrigues. Porto Alegre: Artes Médicas, 1991.

MUNHOZ, Maria Luzia Puglisi. Educação e família numa visão psicopedagógica sistêmica. In: SCOZ, Beatriz Judith Lima et al. (org.). Psicopedagogia: contribuições para a educação pós-moderna. Petrópolis: Vozes, 2004. p. 175-183. 
PARENTE, Sonia Maria. B. Albuquerque; RANÑA, Wagner. Dificuldades de aprendizagem: discussão crítica de um modelo de atendimento. In: SCOZ, Beatriz Judith Lima et al. (org.). Psicopedagogia: o caráter interdisciplinar na formação e atuação profissional, Porto Alegre: Artes Médicas, 1987. p. $48-55$.

PORTELLA, Fabiani Ortiz; HICKEL, Neusa Kern. Psicopedagogia no cotidiano escolar: impasses e descobertas com o ensino de nove anos. Psicopedagogia, São Paulo, v. 27, n. 84, p. 372-384, 2010. Disponível em http://www.revistapsicopedagogia.com.br/detalhes/192/psicopedagogiano-cotidiano-escolar--impasses-e-descobertas-com-o-ensino-de-nove-anos. Acesso em: 20 set. 2020.

RUBINSTEIN, E. A Especificidade do Diagnóstico Psicopedagógico. In: SISTO, F. F. et al. (Org.). Atuação psicopedagógica e aprendizagem escolar. 5. ed. Petrópolis: Vozes, 2000. p. 127-139.

SIGNOR, Rita; CLAESSEN, Mary; LEITÃO, Suze. Interventions for dyslexia in Brazil: a scoping review discussed within the perspective of international best practice. Australian Journal of Learning Difficulties, v. 25, n. 1, p. 1-30, 2020. Disponível em: https://doi.org/10.1080/19404158.2019.1709216. Acesso em: 20 set. 2020.

STEIN, Lilian Milnitsky. TDE - Teste de desempenho escolar: manual para aplicação e interpretação. São Paulo: Casa do psicólogo, 1994.

VISCA, Jorge. Técnicas projetivas psicopedagógicas e pautas gráficas para sua interpretação. Buenos Ayres: Visca e Visca, 2008.

VISCA. Jorge. Clínica psicopedagógica: epistemologia Convergente. Porto Alegre: Artes Médicas, 1987.

WAGNER, A. Família em cena: trama, dramas e transformações. Rio de Janeiro: Vozes, 2002.

WEISS, Maria Lúcia Lemme. Psicopedagogia clínica: uma visão diagnóstica dos problemas de aprendizagem escolar. 7. ed. Rio de Janeiro: DP \& A, 2000.

WEISS, Maria Lúcia Lemme. Vencendo as dificuldades de aprendizagem escolar. Rio de Janeiro: Wak, 2011. 